\title{
Radiofrequency in Clinical Dermatology
}

Jae Min Shin

Jeong Eun Kim

Department of Dermatology, Hanyang University Hospital, Hanyang University College of Medicine, Seoul, Korea

Received October 8, 2013

Revised October 25, 2013

Accepted November 8, 2013

\footnotetext{
Correspondence

Jeong Eun Kim

Department of Dermatology, Hanyang University Hospital, 17 Haengdang-dong, Seongdong-gu,

Seoul 133-792, Korea

Tel: +82-2-2290-8436

Fax: $+82-2-2291-9619$

E-mail: dermakimagmail.com

(C) Korean Society for Laser Medicine and Surgery

(c) This is an open access article distributed under the terms of the Creative Commons Attribution NonCommercial License (http://creativecommons.org/ licenses/by-nc/3.0) which permits unrestricted noncommercial use, distribution, and reproduction in any medium, provided the original work is properly cited.
}

With the increasing interest in anti-aging, the demand for less invasive, non-surgical treatments has increased as well. Radiofrequency (RF) uses electric current instead of optical sources like laser and uses the energy generated from the current to generate heat inside the dermis. The heat, as a result, triggers dermal remodeling to give its anti-aging effects. RF treatment has been used in the field of cosmetic dermatology to improve skin laxities, wrinkles, acne scars, cellulite, and striae distensae since early 2000s, and many a number of previous studies have been published using various medical devices. Based on the layout of the electrodes, the RF treatment comes in two different types, namely the monopolar type and the bipolar type. Recently, the fractional type RF technology has been introduced and used widely. The outcomes so far show that the RF treatment is effective for treating aging skin and scar treatment in mild to moderate degrees of severity. It is one of the effective and safe treatment option and has the advantage of the patient being able to go back to their normal lives faster. In this review, we will examine the fundamental working mechanism of the RF technology and summarize the profound study results in the field.

\section{Key words}

Radiofrequency; Rejuvenation 


\section{INTRODUCTION}

As the interest and demands for cosmetic treatments increase, the tendency of the rejuvenation treatment, which mainly depended on the surgical treatments in the past, is shifting toward less invasive, more effective treatments which allow faster recovery to normal lives. In line with such a tendency, the radiofrequency (RF) treatment is gaining more attention these days. Unlike the laser treatment, the RF treatment uses a new, nonablative technology utilizing electric current for skin rejuvenation. RF gives focal dermal heat damages on the dermis, which results in less damage on the epidermis, reduction in the adverse events, and faster recovery to normal lives. There is a variety of RF technology devices, including monopolar, bipolar, and factional RFs. In this review, we will summarize and compare the most representative studies in the field of RF technology.

\section{HISTORY}

RF energy has been in use with various medical devices. Bovies, which are still widely used in the operation rooms, are one of the most profound examples of RF electrocautery device. In dermatology, RF technology was first introduced for nonablative skin rejuvenation after FDA approved RF treatment for facial wrinkle reductions in early 2000s. And then RF devices were approved for offface treatments in $2006 .{ }^{1}$

\section{RF TECHNOLOGY}

RF devices generate an electric current within an electromagnetic field of $3 \mathrm{kHz}-300 \mathrm{mHz}$ frequency. ${ }^{1-3}$ Once the current reaches the tissue, the current meet the unique resistance of the tissue, which is called the impedance. When the current goes through a tissue with electric resistance, heat is generated, as the electric energy is converted to heat energy. ${ }^{4}$ Such an energy discharge amount can be calculated using the following formula;

$$
\begin{aligned}
& \text { Energy }(\mathrm{J})=I^{2} \times z \times t \\
& \text { Here, } I=\text { current, } z=\text { impedance, } t=\text { time (seconds). }
\end{aligned}
$$

Therefore, it could be said that the amount of the generated energy depends on the energy that flows on the impedance of the target tissue. The subcutaneous fat layer or other places of high resistance can generate a larger amount of energy, thus making it possible for the heat to penetrate even deeper layers. While diffraction, absorption, and scatters cause the loss of a part of energy generated by a laser or other optical devices, RF energy is free from the influence of diffraction, absorption by the chromophores, or other tissue interactions, which, therefore, give no influences on to the epidermal melanin. For this reason it could be used for any skin types, and it is possible to control the depth of the energy's infiltration. ${ }^{2-4}$

The contraindications of RF treatment include the patients with implantable medical devices, such as a pacemaker or a defibrillator. Those with collagen vascular diseases or autoimmune diseases are also contraindicated. In addition, caution is required when using RF treatments for patients who are under radioactive treatment or recurring herpes infections. ${ }^{5,6}$

RF devices come in two different types according to the configurations of electrode, which are the monopolar type and the bipolar type. The monopolar type uses a single electrode tip, which delivers energy to the space between the electrode and the grounding plate. The bipolar type passes electrical current between the two electrodes applied to the skin. The bipolar type penetrates a smaller volume of tissues, and this allows it to work even less energy compared to the monopolar type. The depth of heating depends on the size and geometry of the treatment tip. For the bipolar type, the depth is half of the distance between the two electrodes. This gives the monopolar type of being able to penetrate deeper, but the bipolar type is still superior in terms of the pain it causes.

\section{MONOPOLAR RF}

One of the most representative devices of the monopolar type, the Thermacool system (Thermacool, Thermage, Inc., Hayward, CA) emits an RF energy of 6 $\mathrm{MHz}$, which is delivered onto the skin through a disposable electrode tip. For this type of devices, cryogen is used as the cooling module. Fitzpartrick et al. ${ }^{7}$ conducted a largescale clinical study using the monopolar RF technology and proved its efficacy in treating the periorbital wrinkles. In that study, 86 subjects were treated once with the device, and $83.2 \%$ of the treated patients showed grade 1 or higher improvement of the periorbital wrinkles. In addition, $61.5 \%$ of the patients showed an eyebrow lift of 0.5 $\mathrm{mm}$ or higher in a photographic assessment. As a result of this study, US FDA approved the use of Thermage on the upper face wrinkles in 2002, and the entire face in 2004.

In addition to facial rejuvenation, it could be used for improvement of the acne or acne scars. The most repre- 
sentative treatment mechanism of this technology for these conditions can be the restoration of the activities of sebaceous glands and the recovery of collagen in the dermis. In a clinical study with 22 patients, where 1 to 3 treatment sessions were given to the patients using 65$103 \mathrm{~J} / \mathrm{cm}^{2}$ of energy, $92 \%$ of the patients showed $75 \%$ or more reduction of the number of acne lesions, while $9 \%$ of them showed $25-50 \%$ reduction in lesion counts. In addition to the lesions of acnes, some patients also showed improvements of the acne scars. ${ }^{8}$

The treatment mechanism of the monopolar RF is to generate heat to cause two different effects on collagen. Firstly, the heat disintegrates the hydrogen bonds to change the triple helix structures, resulting in a contraction of collagen. Secondly, the gradual contraction along with wound healing process causes regeneration of collagen. Such changes take place of a period of 2 to 6 months in a slow pace, resulting in a thicker layer of regenerated dermis. ${ }^{9,10}$

Two aspects should be considered when determining which is better between treating in a single session with a higher energy or using less energy over a number of sessions. The first aspect is the 'effect', and the other is the 'side effect' of the treatment. In earlier days, using a higher amount of energy concentrated in a single session was more popular. The biggest problem with this approach was the pain it caused. Further studies afterwards revealed that applying a lower amount of energy over a number of sessions was more helpful for collagen denaturation and the improvement of the skin elasticity. Since then, bigger tips started to be used for the treatment, and the recent trend seems to apply less energy over a number of sessions. ${ }^{10}$

According to the studies of long-term treatment effects of the monopolar RF technology by Suh et al.," in which 8 patients were followed-up for a period of 6 to 7 years, they received 4 sessions of the treatment in average without using any other rejuvenation treatments. The severity of the wrinkles, based on Glogau's wrinkle classification system, maintained the improved status without worsening after treatment. $75 \%$ of the patients were satisfied with the results, and $25 \%$ of the patients were highly satisfied. In the preceding studies, the patients in their 30s to 40s appeared to maintain the best effects, and the maintained effects reduced as the patients grew older. However, in the long-term follow-up study, patients of all ages, including those in their 60s, maintained the effects of the treatment desirably. ${ }^{11,12}$

The authors of the study concluded that, in consideration of the preceding findings that lower-energy/high passes approach was better in terms of the effects of the treatment and the stability of the patients, recommended multiple treatment sessions with approximately 1 year intervals and a higher number of passes in each session. ${ }^{11}$

The most representative of such studies are summarized in Table 1.

\section{BIPOLAR RF}

The biggest difference between the bipolar type and the monopolar type is the 'configuration.' While the monopolar type uses only one electrode placed on the skin and requires a grounding electrode on the other side, the bipolar type RF requires two electrodes to contact the skin and the current to flow from one electrode to another. The electric current, for this reason, could go as deep as half the distance between the two electrodes. The major limitation of this configuration is the depth of penetration. The monopolar device achieves high penetration of the emitted current, which serves as its main advantage and also its major drawback, which is associated with pain. The bipolar configuration is not as penetrating but provides more-controlled distribution of energy and less pain. In order to maximize the benefits of the bipolar RF, the electro-optical synergy (ELOS) technology where the light and laser are used together could be applied, or the FACES technology (functional aspiration controlled electrothermal stimulation) could be used, where vacuum is used to maximize the depth of penetration. ${ }^{4,19,20}$

The basic mechanism of treatment is similar to that of the monopolar type, where the emitted energy causes the contraction of the collagen in dermis, the inflammatory process, and the contraction of the fibrous septa.

Table 1 shows a summary of various studies using the bipolar RF technology.

In the ELOS system, which was developed by Syneron (Syneron Medical Ltd., Yokneam, Israel), the IPL, diode laser, or other light energy could be used to pre-heat the target tissue through photothermolysis and to lower the impendence of the tissue and, as a result, making it easier for the RF energy to penetrate the tissues. In addition, the optical components can be used for a direct reaction with fibroblasts, blood vessels, and the pigments in the epidermis. Some of the most well-known examples of ELOS technology include Aurora SR, which uses both of RF and IPL technology, Polaris WR, which combines RF with $900 \mathrm{~nm}$ diode laser, VelaSmooth which combines RF and infrared light of 700-2,000 nm with a suction system. ${ }^{12-27}$ Furthermore, each of the combined hand 


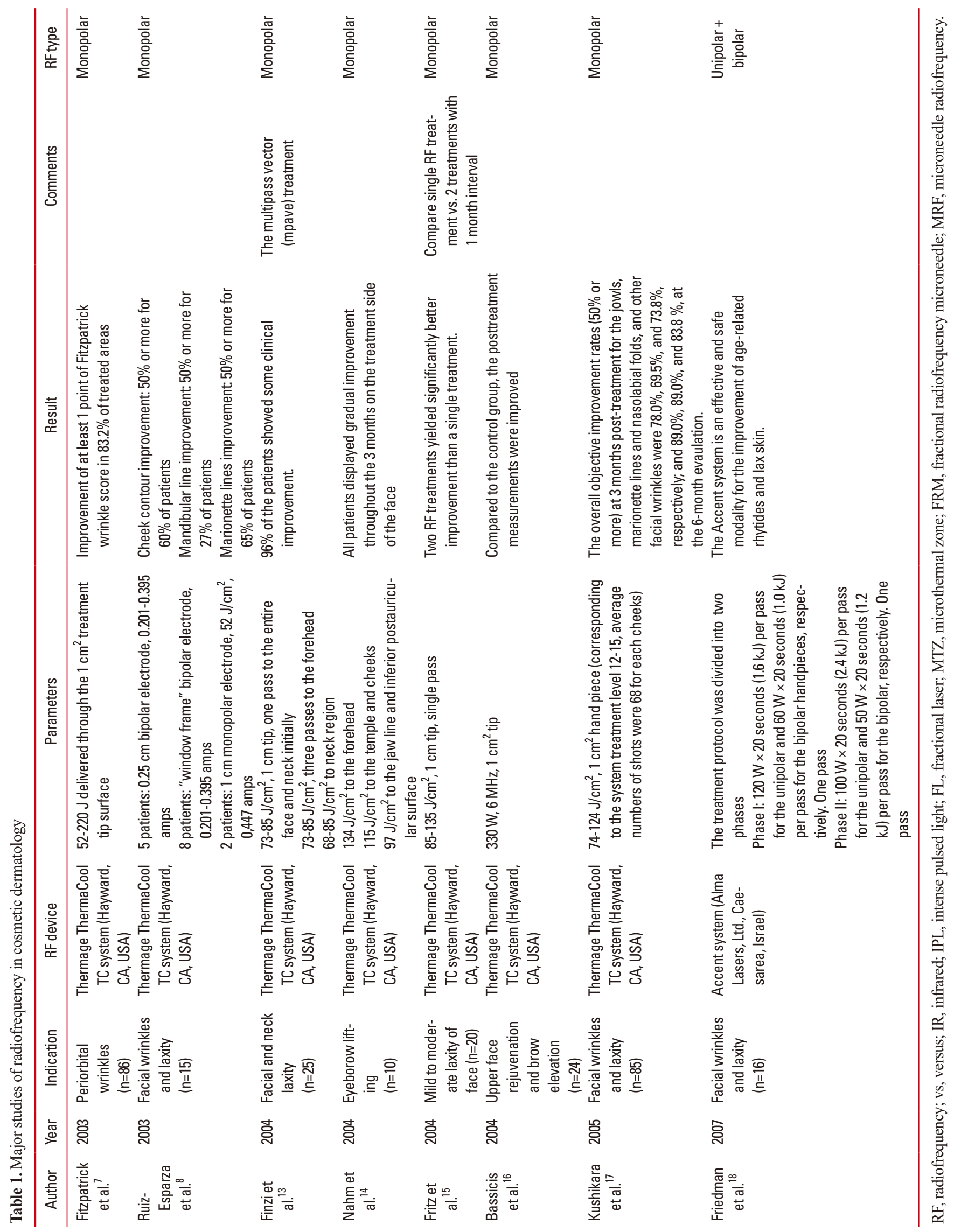




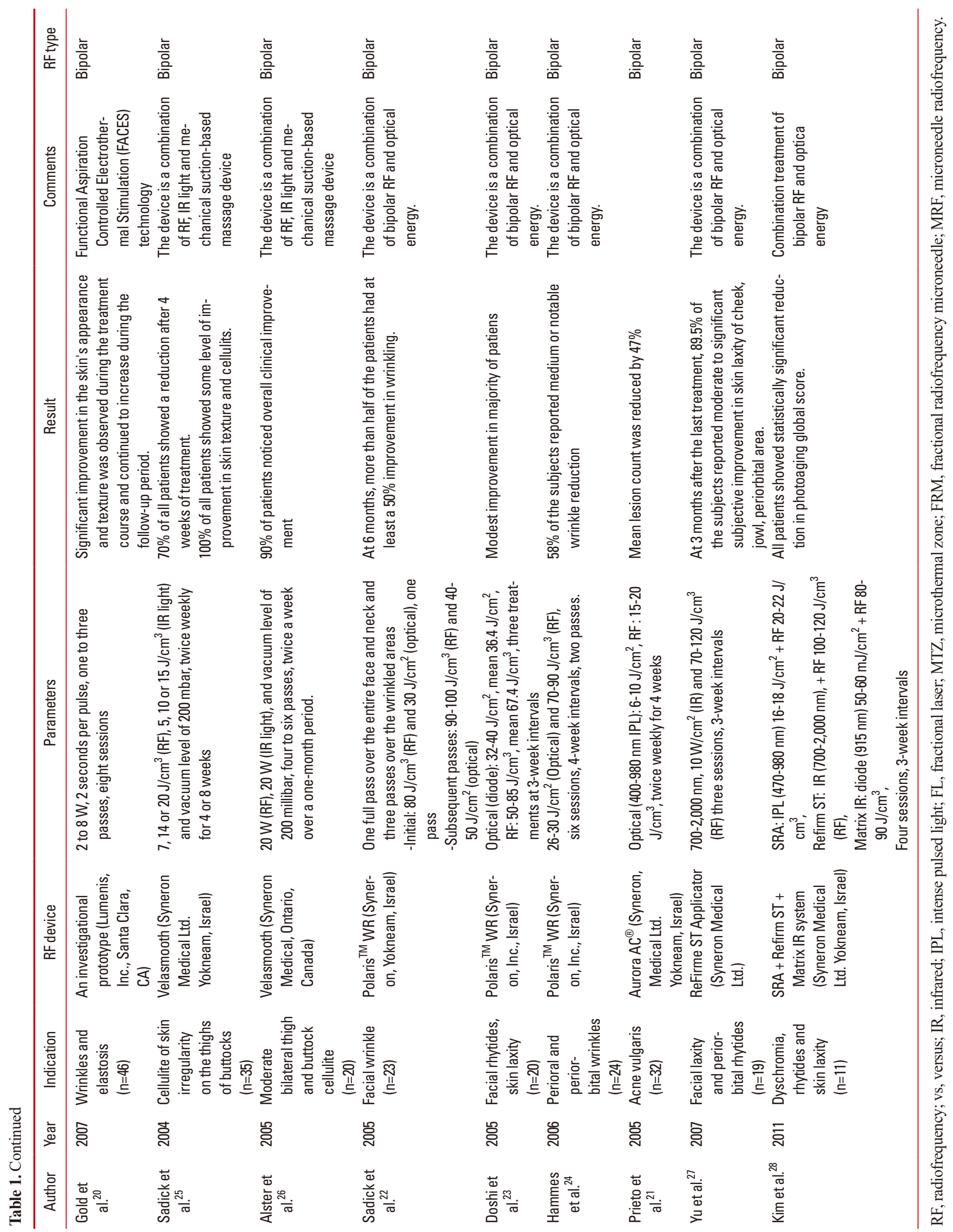



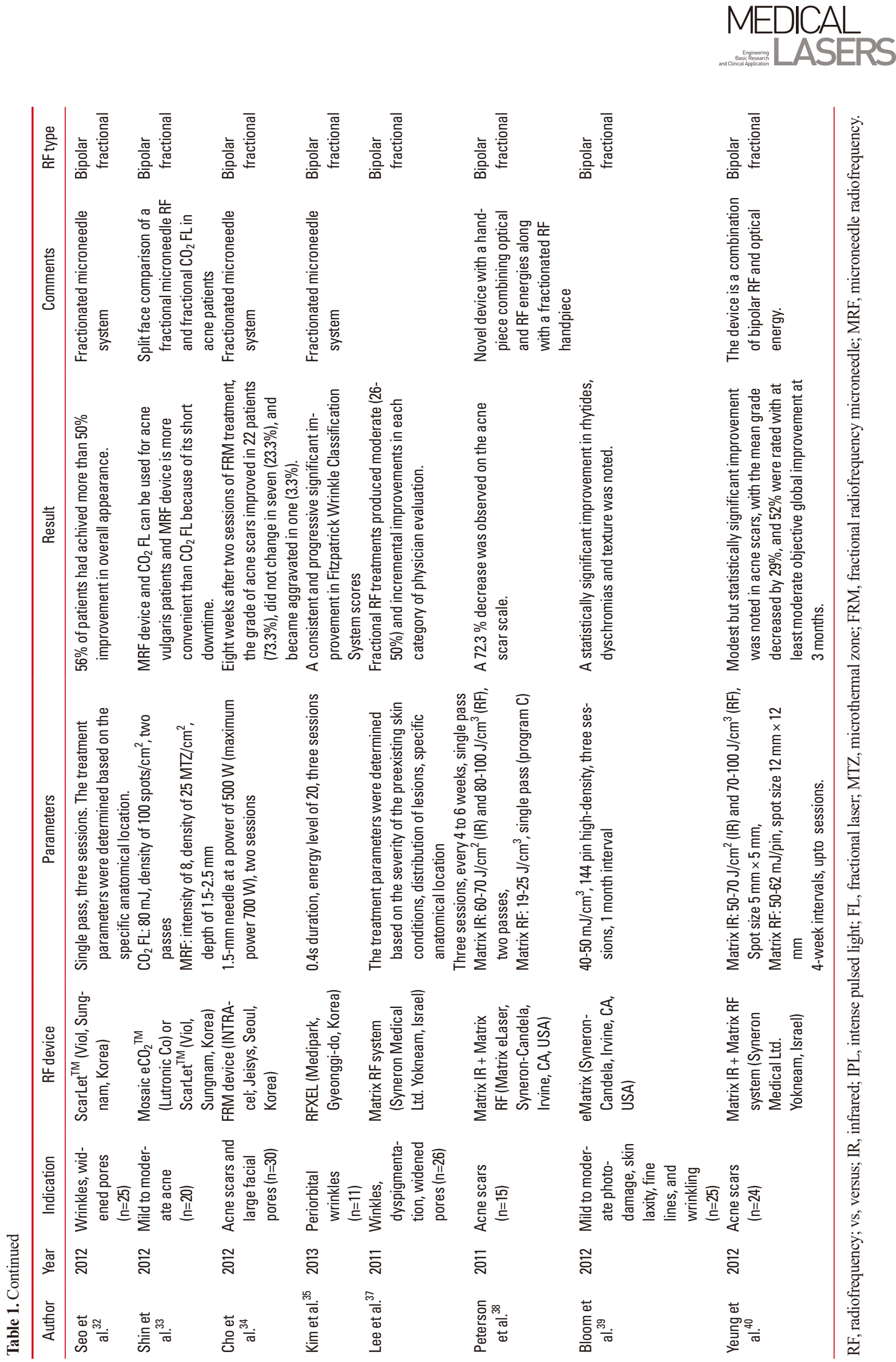
pieces could be selected and operated on a basic platform of single RF device according to the physician's choice. With using three handpieces in a serial order (RF + IPL, RF + infrared light, RF + diode laser), we previously achieved the improvement of superficial dyschromia, erythema and wrinkles in a single treatment. The measurements proved that the melanin index (MI) and erythema index (EI) decreased, and the cutometer measurements also showed an improvement of the elasticity of the skin. ${ }^{28}$

The devices using FACES technology (Aluma System Lumenis, Inc., Santa Clara, CAl is composed of an RF generator, handpiece, and tips with two horizontal electrodes. This device creates a vacuum by means of suction. In a study with 46 patients who received treatments over 5 sessions in 1 to 2 weeks' intervals showed statistically significant improvements in terms of the facial wrinkles and the skin elasticity. They also showed $50 \%$ improvements in the treatment of striae distensae..$^{20,29}$

The most representative of the studies using bipolar RF are summarized in Table 1.

\section{FRACTIONAL RF}

The fractional RF technology is a newly developed form of RF treatment, which is divided in two different types in the mode of energy delivery. One of them is an RF system using a microneedle, while the other is a system which is represented by the Matrix RF, using the bipolar RF to trigger a partial epidermal ablation. In as study using Renesis (Prmavea Medical, Inc. Fermont, CA), the generation of the controlled RF thermal zone (RFTZs) was confirmed. The advantage of this system is that, it can deliver the fractional RF energy using a microneedle with protection of the adnexal structures. ${ }^{30}$

In a study that evaluated the histological changes after fractional RF treatment, it was shown that molecular changes up to 10 weeks after the treatment, consecutive inflammatory reactions were observed starting from day 2 after treatment, which triggered the wound healing process and RFTZs were replaced by new collage as a result of the heat shock protein generation. Then, neoelastogenesis occurred to result in dermal remodeling. $^{31}$

Based on several studies, it could be concluded that the fractional microneedle RF technology was effective in treating wrinkles and the scars of acnes. ${ }^{32-35}$

Matrix RF system (Syneron Medical, Ltd) is an RF treatment equipment that aligns the multielectrode pins in a pair to induce partial epidermal ablation. The nonhomogenous thermal wounds on the skin are directly delivered to the reticular dermis, while other areas than the target area are maintained intact, resulting in faster recovery from the wounds. The histological finding confirmed that the demarcated zones, which were identified by ablation, coagulation, necrosis, and subnecrosis, were generated to $450 \mathrm{um}$ in depth. Along with that, higher energy and a low coverage rate resulted in good clinical outcomes while causing less pain to the patients. ${ }^{36}$

In a study with Asian population, the technology showed good clinical results on wrinkles, skin texture, or pores without post-inflammatory hyperpigmentation. In addition, the technology was also effective against acnes or acne scars. ${ }^{37-40}$ In the study by the author, clinical improvements were observed after 4 sessions in terms of the depressed scars and enlarged pores. In that study, all of $\mathrm{MI}, \mathrm{El}$, and cutometer measurements showed significant improvements after treatment. In addition, histological observations showed that procollagen I, III, and elastin increased after treatment, causing less hindrance in daily lives due to shorter downtime caused by erythema or crust and less pain (unpublished data).

\section{CONCLUSION}

The development in RF technology for skin rejuvenation allowed a large variety of non-surgical anti-aging treatment. Up until now, RF treatments have been used for skin laxity, rhytides, acne vulgaris, scar, and cellulites. Most of the results of the treatments were positive, but the groups with the best chance of the positive results were the patients who showed the early signs of aging with mild to moderate degrees. In the meantime, the patients with profound skin fold or laxity required more invasive treatments or combined treatments. For this reason, treatments of these days usually combine other treatments, such as laser, botulinum toxin, or fillers, etc., to achieve the maximum effects with the minimum downtime. The RF will remain as the promising alternative treatment for ablative and nonablative laser and light based treatments. Further studies would be necessary to identify the ideal treatment parameters lenergy level and the number of passes) and the treatment intervals, in a comparative study between different types of RF technologies and on new indications, as well.

\section{REFERENCES}

1. Alexiades-Armenakas M, Dover JS, Arndt KA. Unipolar versus bipolar radiofrequency treatment of rhytides and laxity using 
a mobile painless delivery method. Lasers Surg Med 2008;40: 446-53.

2. el-Domyati M, el-Ammawi TS, Medhat W, Moawad O, Brennan D. Mahoney MG, et al. Radiofrequency facial rejuvenation: evidence-based effect. J Am Acad Dermatol 2011;64:524-35.

3. Elsaie ML. Cutaneous remodeling and photorejuvenation using radiofrequency devices. Indian J Dermatol 2009;54:201-5.

4. Alster TS, Lupton JR. Nonablative cutaneous remodeling using radiofrequency devices. Clin Dermatol 2007;25:487-91.

5. Abraham MT, Vic Ross E. Current concepts in nonablative radiofrequency rejuvenation of the lower face and neck. Facial Plast Surg 2005;21:65-73.

6. Ruiz-Esparza J. Nonablative radiofrequency for facial and neck rejuvenation. A faster, safer, and less painful procedure based on concentrating the heat in key areas: the ThermaLift concept. J Cosmet Dermatol 2006;5:68-75.

7. Fitzpatrick R, Geronemus R, Goldberg D, Kaminer M, Kilmer S, Ruiz-Esparza J. Multicenter study of noninvasive radiofrequency for periorbital tissue tightening. Lasers Surg Med 2003;33:23242.

8. Ruiz-Esparza J, Gomez JB. Nonablative radiofrequency for active acne vulgaris: the use of deep dermal heat in the treatment of moderate to severe active acne vulgaris (thermotherapy): a report of 22 patients. Dermatol Surg 2003;29:333-9; discussion 339.

9. Jacob Cl, Kaminer MS. Skin tightening with radiofrequency. In: Goldberg DJ, editor. Procedures in Cosmetic Dermatology Series: Laser and Lights. Philadelphia: Elservier Saunders; 2005. p. 43-60.

10. Zelickson BD, Kist D, Bernstein E, Brown DB, Ksenzenko S, Burns J, et al. Histological and ultrastructural evaluation of the effects of a radiofrequency-based nonablative dermal remodeling device: a pilot study. Arch Dermatol 2004;140:2049.

11. Suh DH, Lee SJ, Ryou JH, Son HC, Kim HJ, Kim HS. Monopolar radiofrequency treatment in Asian skin: do multiple RF treatments over time have beneficial effects? An observational report with long-term follow-up in eight patients. Dermatol Surg 2013; 39:670-2.

12. Suh DH, Chang KY, Ryou JH, Lee SJ, Kim HS. Monopolar radiofrequency treatment in Asian skin: a questionnaire-based study. J Cosmet Laser Ther 2011;13:126-9.

13. Finzi E, Spangler A. Multipass vector (mpave) technique with nonablative radiofrequency to treat facial and neck laxity. Dermatol Surg 2005;31:916-22.

14. Nahm WK, Su T, Rotunda AM, Moy RL. Objective changes in brow position, superior palpebral crease, peak angle of the eyebrow, and jowl surface area after volumetric radiofrequency treatments to half of the face. Dermatol Surg 2004;30:922-8; discussion 928 .
15. Fritz M, Counters JT, Zelickson BD. Radiofrequency treatment for middle and lower face laxity. Arch Facial Plast Surg 2004;6: 370-3.

16. Bassichis BA, Dayan S, Thomas JR. Use of a nonablative radiofrequency device to rejuvenate the upper one-third of the face. Otolaryngol Head Neck Surg 2004;130:397-406.

17. Kushikata N, Negishi K, Tezuka Y, Takeuchi K, Wakamatsu S. Non-ablative skin tightening with radiofrequency in Asian skin. Lasers Surg Med 2005;36:92-7.

18. Friedman DJ, Gilead LT. The use of hybrid radiofrequency device for the treatment of rhytides and lax skin. Dermatol Surg 2007;33:543-51.

19. Atiyeh BS, Dibo SA. Nonsurgical nonablative treatment of aging skin: radiofrequency technologies between aggressive marketing and evidence-based efficacy. Aesthetic Plast Surg 2009;33:283-94.

20. Gold MH, Goldman MP, Rao J, Carcamo AS, Ehrlich M. Treatment of wrinkles and elastosis using vacuum-assisted bipolar radiofrequency heating of the dermis. Dermatol Surg 2007;33: 300-9.

21. Prieto VG, Zhang PS, Sadick NS. Evaluation of pulsed light and radiofrequency combined for the treatment of acne vulgaris with histologic analysis of facial skin biopsies. J Cosmet Laser Ther 2005;7:63-8.

22. Sadick NS, Trelles MA. Nonablative wrinkle treatment of the face and neck using a combined diode laser and radiofrequency technology. Dermatol Surg 2005;31:1695-9.

23. Doshi SN, Alster TS. Combination radiofrequency and diode laser for treatment of facial rhytides and skin laxity. J Cosmet Laser Ther 2005;7:11-5.

24. Hammes S, Greve B, Raulin C. Electro-optical synergy (ELOS) technology for nonablative skin rejuvenation: a preliminary prospective study. J Eur Acad Dermatol Venereol 2006;20:10705.

25. Sadick NS, Mulholland RS. A prospective clinical study to evaluate the efficacy and safety of cellulite treatment using the combination of optical and RF energies for subcutaneous tissue heating. J Cosmet Laser Ther 2004;6:187-90.

26. Alster TS, Tanzi EL. Cellulite treatment using a novel combination radiofrequency, infrared light, and mechanical tissue manipulation device. J Cosmet Laser Ther 2005;7:81-5.

27. Yu CS, Yeung CK, Shek SY, Tse RK, Kono T, Chan HH. Combined infrared light and bipolar radiofrequency for skin tightening in Asians. Lasers Surg Med 2007:39:471-5.

28. Kim JE, Chang S, Won CH, Kim CH, Park KH, Choi JH, et al. Combination treatment using bipolar radiofrequency-based intense pulsed light, infrared light and diode laser enhanced clinical effectiveness and histological dermal remodeling in Asian photoaged skin. Dermatol Surg 2012;38:68-76.

29. Montesi G, Calvieri S, Balzani A, Gold MH. Bipolar radiofrequency 
in the treatment of dermatologic imperfections: clinicopathological and immunohistochemical aspects. J Drugs Dermatol 2007;6: 890-6.

30. Hantash BM, Renton B, Berkowitz RL, Stridde BC, Newman J. Pilot clinical study of a novel minimally invasive bipolar microneedle radiofrequency device. Lasers Surg Med 2009;41:87-95.

31. Hantash BM, Ubeid AA, Chang H, Kafi R, Renton B. Bipolar fractional radiofrequency treatment induces neoelastogenesis and neocollagenesis. Lasers Surg Med 2009;41:1-9.

32. Seo KY, Yoon MS, Kim DH, Lee HJ. Skin rejuvenation by microneedle fractional radiofrequency treatment in Asian skin; clinical and histological analysis. Lasers Surg Med 2012;44:631-6.

33. Shin JU, Lee SH, Jung JY, Lee JH. A split-face comparison of a fractional microneedle radiofrequency device and fractional carbon dioxide laser therapy in acne patients. J Cosmet Laser Ther 2012;14:212-7.

34. Cho SI, Chung BY, Choi MG, Baek JH, Cho HJ, Park CW, et al. Evaluation of the clinical efficacy of fractional radiofrequency microneedle treatment in acne scars and large facial pores. Dermatol Surg 2012;38:1017-24.
35. Kim JK, Roh MR, Park GH, Kim YJ, Jeon IK, Chang SE. Fractionated microneedle radiofrequency for the treatment of periorbital wrinkles. J Dermatol 2013;40:172-6.

36. Hruza G, Taub AF, Collier SL, Mulholland SR. Skin rejuvenation and wrinkle reduction using a fractional radiofrequency system. J Drugs Dermatol 2009;8:259-65.

37. Lee HS, Lee DH, Won CH, Chang HW, Kwon HH, Kim KH, et al. Fractional rejuvenation using a novel bipolar radiofrequency system in Asian skin. Dermatol Surg 2011;37:1611-9.

38. Peterson JD, Palm MD, Kiripolsky MG, Guiha IC, Goldman MP. Evaluation of the effect of fractional laser with radiofrequency and fractionated radiofrequency on the improvement of acne scars. Dermatol Surg 2011;37:1260-7.

39. Bloom BS, Emer J, Goldberg DJ. Assessment of safety and efficacy of a bipolar fractionated radiofrequency device in the treatment of photodamaged skin. J Cosmet Laser Ther 2012; 14:208-11.

40. Yeung CK, Chan NP, Shek SY, Chan HH. Evaluation of combined fractional radiofrequency and fractional laser treatment for acne scars in Asians. Lasers Surg Med 2012;44:622-30. 